\title{
Dünnschichtchromatographische Bestimmung von 3-Methoxy-4-hydroxymandelsäure im Harn
}

\author{
Von H. Strobach \\ Aus dem Pharmakologischen Institut der Universität Düsseldorf (Direktor: Prof. Dr. med. K. Grceff)
}

(Eingegangen am 17. August 1966)

In Aule hnung an das Verfahren von Scrimid wird eine Methode zur dünnschichtchromatographischen Bestimmung der Vanillinmandelsäureausscheidung im Harn entwickelt. Dabei wird die Vanillinmandelsäure (VMS) mit Essigester extrahiert und der Extrakt dünnschichtchromatographisch auf Kieselgel G aufgetrennt. Die VMS wird durch Besprühen der Chromatogramme mit diazotiertem pNitroanilin sichtbar gemacht, mit Pufferlösung eluiert und spektralphotometrisch bestimmt.

Die Normalwerte für die VMS-Ausscheidung lagen in unseren Versuchen zwischen 0,9 und $8,6 \mathrm{mg} / 24 \mathrm{Stdn}$. und betrugen im Mittel 4,1 mg. Bei 12 Patienten mit Phaeochromocytom oder Neuroblastom wurden Werte von 10,8-142,3 mg VMS/24 Stdn. beobachtet.

A procedure, which depends upon Scrmid's method, was developed for the thin layer chromatographic determination of the urinary excretion of vanillylmandelic acid (VMA). The acid is extracted with ethyl acetate and the extract separated by thin layer chromatography on Kieselgel G. VMA is located by spraying with diazotised p-nitroaniline, eluted with buffer and determined spectrophotometrically. In our experiments, the normal values for VMA excretion lay between 0.9 and $8.6 \mathrm{mg} / 24 \mathrm{hr}$. with an average of $4.1 \mathrm{mg}$. In 12 patients with phaeochromocytoma or neuroblastoma, values of $10.8-142.3 \mathrm{mg}$. VMA/24 hr. were observed.

Seit Armstrong und Mitarbeiter (1,2) 3-Methoxy-4 hydroxymandelsäure (Vanillinmandelsäure, „VMS“) als Metaboliten des Noradrenalinstoffwechsels im Harn nachweisen konnten, sind zahlreiche Untersuchungen über die Ausscheidung dieser Verbindung im Harn durchgeführt worden. VMS entsteht durch Methylierung des Adrenalins bzw. des Noradrenalins in C-3-Stellung (3-6) und oxydativer Desaminierung der entstandenen O-Methylderivate $(7,8)$. VMS ist also das gemeinsame Abbauprodukt des Adrenalins und Noradrenalins.

Adrenalin und Noradrenalin werden nach i. v. Infusion zu rund $4 \%$ der applizierten Dosis in unveränderter Form ausgeschieden. Rund $20-40 \%$ finden sich im Harn als O-Methyladrenalin bzw. O-Methylnoradrenalin wieder, der Rest vorwiegend als VMS $(9,10)$. Nach Armstrong (2) scheiden Gesunde $1,5-3 \mathrm{mg}$ VMS in 24 Stdn. aus. Von anderen Autoren durchgeführte Untersuchungen führten $\mathrm{zu}$ ähnlichen Ergebnissen (11-21).

Bei den bisher beschriebenen Bestimmungsverfahren wird die Vanillinmañdelsäure nach Abtrennung durch Papierelektrophorese (14, 16, 17), Papierchromatographie $(11,12,18)$ oder Dünnschichtchromatographie (19) kolorimetrisch bestimmt. Nach anderen Methoden wird VMS zu Vanillin umgesetzt, das mit Indol und Phosphorsäure einen kolorimetrisch bestimmbaren Farbkomplex liefert (13). Nach BRUNJES (22) wird VMS durch Perjodat oxydiert und UV-spektrophotometrisch bestimmt.

\section{Methodik}

\section{Prinzip}

Vanillinmandelsäure wird aus dem angesäuerten, mit Kochsalz gesättigten Harn mit Essigester extrahiert. Die organische Phase wird abgetrennt, eingedampft und der Rückstand mit 60-proz. Äthanol aufgenommen. Die Vanillinmandelsäure wird dünnschichtchromatographisch auf Kieselgel $\mathrm{G}$ mit Essigester-Isopropanol-konz. Ammoniak (1:2:1) als Fließmittel abgetrennt. Das noch feuchte Chromatogramm wird mit diazotiertem pNitroanilin besprüht und kurze Zeit mit Ammoniak be- handelt. Die violettrote VMS-Bande wird noch feucht abgetragen und in Borat-Citratpuffer ( $\mathrm{pH} 9,5)$ gebracht. Dabei werden sowohl der gebildete Farbstoff wie nicht umgesetzte Vanillinmandelsäure quantitativ eluiert. Nach Abzentrifugieren vom Kieselgel wird der Überstand mit verdünntem Sprühreagenz versetzt, das mit unverändert aus der Schicht eluierter Vanillinmandelsäure reagiert. Die Messung erfolgt bei $510 \mathrm{~m} \mu$ gegen einen Reagenzienblindwert.

Reagenzien

1.VMS-Standard $(1 \mathrm{mg} / \mathrm{ml}): 100 \mathrm{mg}$ VMS werden in $100 \mathrm{ml}$ $0,01 \mathrm{~N} \mathrm{HCl}$ gelöst. Standardlösungen niedriger Konzentration stellt man durch entsprechendes Verdünnen mit $0,01 \mathrm{~N} \mathrm{HCl} \mathrm{her.}$

2. Natriumchlorid p.a.

3. Natriumsulfat p. a. wasserfrei

4. konz. Salzsäure p. a.

5. Essigester p. a.

6. 60-proz. Äthanol

7. 5-proz. Ammoniumsulfamat

8. $0,01 \mathrm{~N}$ Salzsäure

9. Borat-Citratpuffer $p H$ 9,5:3,54 g Borsäure und 7,00 g Citronensäure werden in $900 \mathrm{~m} l$ Wasser gelöst. Sodann fügt man unter ständigem Rühren $13,70 \mathrm{~g}$ festes Natriumhydroxyd zu. Sobald sich alles gelöst hat, versetzt man langsam mit $2,23 \mathrm{~m} / 85$-proz. Phosphorsäure und schließlich unter Rühren und Kontrolle des $\mathrm{pH}-$ Wertes mit dem $\mathrm{pH}-$ Meter tropfenweise mit soviel konz. Salzsäure, bis der $\mathrm{pH}$-Wert genau 9,5 beträgt. Man füllt auf $1000 \mathrm{~m} l$ auf und kontrolliert den $\mathrm{pH}$-Wert erneut.

10. p-Nitroanilin-Stammlösung: $100 \mathrm{mg}$ p-Nitroanilin werden unter Rühren in $98 \mathrm{~m} l$ Wasser und $2 \mathrm{~m} l$ konz. Salzsäure gelöst.

11. Natriumnitrit-Stammlösung: $200 \mathrm{mg}$ Natriumnitrit werden in $100 \mathrm{ml}$ Wasser gelöst.

12. Sprübreagenz: Man vereinigt je nach Bedarf gleiche Volumina der eisgekühlten Lösungen 10 und 11 und hält die Mischung etwa 20 Min. im Kühlschrank. Anschließend versetzt man das Reagenz zur Zerstörung überschüssigen Nitrits mit $1 / 20$ dcs Gesamtvolumens 5-proz. Ammoniumsulfamatlösung und schüttelt um, bis der gröi3te Teil des sich entwickelnden Stickstoffs entwichen ist.

13. Verdünntes Spriïreagenz: Man verdünnt $2 \mathrm{ml} l$ der Lösung 12 mit $8 \mathrm{~m} l$ Wasser. Lösung 12 und 13 sind jedesmal vor Gebrauch frisch herzustellen.

Aufstellung der Eichkurve

Reiner Standard: 0,1-1,0 ml VMS-Standard (entsprechend 1-23 $\mu \mathrm{g}$ VMS) werden mit $0,01 \mathrm{~N} \mathrm{HCl}$ auf $1 \mathrm{~m} l$ aufgefüllt und mit $0,3 \mathrm{~m} l$ Reagenz 13 versetzt. Man gibt $6 \mathrm{~m} l$ Borat-Citratpuffer $(\mathrm{pH}$ 9,5) zu und schüttelt um. Nach $10 \mathrm{Min}$. wird die Extinktion bei 
$510 \mathrm{~m} \mu$ gegen einen Blindwert gemessen, der auf gleiche Weise wie die Proben jedoch ohne Zusatz von VMS angesetzt wurde. Im angegebenen Bereich verläuft die Extinktionskurve etwa linear. Der Quotient: Extinktion/ $\mu$ g VMS beträgt im Mittel 0,0417, die Fehlerbreite der Bestimmung $\pm 2,9 \%$. Der gebildete Farbstoff ist stabil; die Extinktion ändert sich im Verlauf von 2 Stdn. nicht merklich.

Cbromatographie: $1-23 \mu \mathrm{g}$ VMS werden in $8 \mathrm{~cm}$ breiten Streifen auf $0,25 \mathrm{~mm}$ dicke Schichten von Kieselgel $G$ aufgetragen. Die Chromatogramme werden mit Essigester-Isopropanol-konz. Ammoniak (1:2:1) entwickelt und noch feucht mit Reagenz 12 be= sprüht. Man bringt die Platten 15-20 Sek. in eine mit etwa $20 \mathrm{ml}$ konz. Ammoniak beschickte Kammer, trägt die angefärbten Zonen der noch feuchten Schicht ab und eluiert mit $6 \mathrm{~m} l$ Borat-Citratpuffer ( $\mathrm{pH} 9,5)$. Man schüttelt 30 Sek. und zentrifugiert 15 Min. bei 4000 U./Min. Der Úberstand wird in ein zweites Röhrchen mit $1 \mathrm{ml} 0,01 \mathrm{~N} \mathrm{HCl}$ und $0,3 \mathrm{~m} l$ verdünntem Sprühreagenz dekantiert und umgeschüttelt. Nach 10 Min. wird bei $510 \mathrm{~m} \mu$ gegen einen Reagenzienleerwert gemessen. Bei Konzentrationen von mehr als $1 \mu \mathrm{g}$ VMS verläuft die erhaltene Eichkurve linear. Der Quotient: Extinktion/ $\mu \mathrm{g}$ VMS liegt mit 0,0380 etwa $10 \%$ niedriger als bei direkter Bestimmung der VMS ohne vorhergehende Chromatographie. Die Fehlerbreite der Methode beträgt $\pm 4,2 \%$ (Abb. 1).

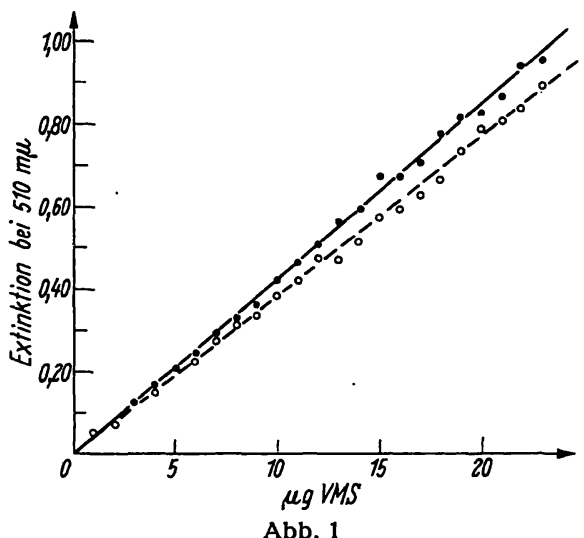

Die durchgezogene Linie stellt die mit reiner Vanillinmandelsäure erhaltene Eichkurve dar. Die gestrichelte Gerade wurde nach chromatoSchicht gewonnen (ZEISS-Spektralphotometer PM Q II. Schichtdicke: $20 \mathrm{~mm}$; Wellenlänge : $510 \mathrm{~m} \mu$ )

\section{Aufarbeitung der Harnproben}

$20 \mathrm{ml}$ des mit Schwefelsäure auf pH 3 angesäuerten 24 Stdn.-Sammelharnes werden mit $7 \mathrm{~g}$ Natriumchlorid versetzt und umgeschüttelt. Anschließend wird die Probe mit konz. $\mathrm{HCl}$ auf $\mathrm{pH} 1$ angesäuert und dreimal je 2 Min. mit 40, 30 und $20 \mathrm{ml}$ Essigester ausgeschüttelt. Die organischen Phasen werden vereinigt, unter gelegentlichem Umschütteln 20 Min. über $10 \mathrm{~g}$ Natriumsulfat getrocknet und filtriert. Das hinterbleibende Natriumsulfat wird mit weiteren $10 \mathrm{ml}$ Essigester versetzt, umgeschüttelt und nach etwa $5 \mathrm{Min}$. erneut filtriert. Die vereinigten Filtrate werden auf zwei Erlenmeyerkolben von $100 \mathrm{~m} l$ Inhalt verteilt und in einem beheizbaren Vakuumexsikkator bei höchstens $40^{\circ}$ auf etwa $5-10 \mathrm{~m} l$ eingeengt. Die hinterbleibende Lösung wird in einen $25 \mathrm{ml}$ Erlenmeyerkolben überführt und zur Trockne eingedampft. Der Rückstand wird nach völligem Abkühlen des Kölbchens mit $1 \mathrm{ml}$ 60-proz. Äthanol aufgenommen.

\section{Dünnschichtchromatographische Trennung}

Die dünnschichtchromatographische Trennung der erhaltenen Extrakte erfolgt auf Schichten von Kieselgel G (Schichtdicke 0,25 mm). Die Schicht wird durch senk- rechte Striche in 2 Bahnen von $8 \mathrm{~cm}$ und zwei Bahnen von $1 \mathrm{~cm}$ Breite zerlegt. Auf eine $8 \mathrm{~cm}-B a h n$ trägt man je nach ausgeschiedener 24 Stdn.-Menge und erwarteter VMS-Konzentration 0,05-0,20 ml Extrakt in Streifenform auf. Etwa 1/8 der Menge wird - ebenfalls in Streifenform - auf eine der als Leitchromatogramm dienenden $1 \mathrm{~cm}-B$ ahnen aufgetragen. Auf das Leitchromatogramm trägt man noch zusätzlich $0,002 \mathrm{ml}$ einer Lösung von $500 \mu \mathrm{g} V M S / \mathrm{m} l 0,01 \mathrm{~N} \mathrm{HCl}$ (entsprechend $1 \mu \mathrm{g}$ VMS) als Vergleichssubstanz auf. Außer bei abnorm hoher Ausscheidung hebt sich die aufgetragene VMS im fertigen Chromatogramm stets als intensiv gefärbte Zone $\mathrm{ab}$, so daß eine Verwechslung mit anderen, ähnlich gefärbten Substanzen im Chromatogramm nicht möglich ist. Auf die verbleibenden zwei Bahnen kann ein zweiter zu untersuchender Extrakt aufgetragen werden. Die Breite der aufgetragenen Streifen sollte 3-5 mm nicht überschreiten. Je breiter die Streifen an der Startlinie sind, desto breiter und unschärfer werden auch die Banden der einzelnen Substanzen im besprühten Chromatogramm.

Man entwickelt mit Essigester-Isopropanol-konz. Ammoniak (1:2:1) als Fließmittel ohne Kammerübersättigung, wobei in jeder Kammer nur ein Chromatogramm entwickelt werden darf. Die Laufstrecke beträgt $15 \mathrm{~cm}$, die Trennzeit etwa 3-4 Stdn.

\section{Bestimmung der abgetrennten VMS}

Man nimmt die entwickelte Platte aus der Kammer, streift die Schicht unterhalb der Startlinie ab und behandelt noch feucht mit unverdünntem Sprühreagenz. Es ist darauf zu achten, daß die Schicht gleichmäßig feucht bleibt, ohne daß jedoch Tropfen daran abfließen. Man besprüht dazu zweckmäßig in kurzen Intervallen und wartet bis das aufgesprühte Reagenz in die Schicht eingedrungen ist. Die Vanillinmandelsäure tritt als rotvioletter Streifen in Erscheinung und ist anhand der auf dem Leitchromatogramm aufgetragenen authentischen Substanz leicht zu lokalisieren. Nach dem Besprühen wird die Platte $15-20$ Sek. in eine mit etwa $20 \mathrm{ml}$ konz. Ammoniak beschickte Kammer gebracht. Der VMS-Streifen wird markiert, die Schicht noch feucht abgekratzt und in ein $15 \mathrm{ml}$ Z̈entrifugenröhrchen mit $6 \mathrm{ml}$ Borat-Citratpuffer $(\mathrm{pH} 9,5)$ gebracht. Man schüttelt etwa $30 \mathrm{Sek}$. und zentrifugiert $15 \mathrm{Min}$. bei 4000 U./Min. Der ganze Arbeitsgang vom Herausnehmen der Platte aus der Kammer bis zum Einbringen der abgekratzten Schicht in die Pufferlösung darf nicht mehr als 3-4 Min. beanspruchen. Aritrocknen der Schicht vor oder nach dem Besprühen führt zu fehlerhaften (zu niẹdrigen) Werten.

Der nach dem Zentrifugienren erhaltene Überstand wird vorsichtig, ohne daß dabei der Niederschlag aufgewirbelt wird, in ein zweites Röhrchen mit $1 \mathrm{ml} 0,01 \mathrm{~N} \mathrm{HCl}$ und $0,3 \mathrm{ml}$ verdünntem Sprühreagenz umgefüllt. Man schüttelt kurz um und mißt nach $10 \mathrm{Min}$. gegen einen Reagenzienblindwert bei einer Wellenlänge von $510 \mathrm{~m} \mu$. Die in 24 Stdn. ausgeschiedene VMS wird nach folgender Formel berechnet: 


$$
\mathrm{VMS}=\frac{\mathrm{H} \times \mathrm{E}}{20 \times \mathrm{e} \times \mathrm{F} \times 1000}
$$

Dabei sind: $\quad$ VMS $=$ mg VMS/24 Stdn.

$$
\begin{aligned}
& \mathrm{H}=24 \text { Stdn.-Ham in } \mathrm{ml} \\
& \mathrm{E}=\text { gemessene Extinktion } \\
& \mathrm{e} \quad=\text { aufgetragene Extraktmenge in } \mathrm{ml} \\
& \mathrm{F} \quad=\text { Extinktion } / \mu \mathrm{g} \text { VMS }=0,038
\end{aligned}
$$

Reproduzierbarkeit und Ausbeute

8 Proben des gleichen Harnes wurden in der beschriebenen Weise getrennt aufgearbeitet und der VMS-Gehalt bestimmt. Die Standardabweichung betrug $\pm 5,5 \%$. In einem weiteren Versuch wurden 8 von 10 Proben des gleichen Harnes $60-200 \mu \mathrm{g}$ VMS zugesetzt und alle 10 Proben in der beschriebenen Weise verarbeitet. Der in der Doppelbestimmung ermittelte Vanillinmandelsäuregehalt des ohne Zusätze verarbeiteten Harnes wurde vom gefundenen Gesamtgehalt abgezogen. Dabei konnten durchschnittlich $99 \%$ der zugesetzten Vanillinmandelsäure wiedergefunden werden $(83,5-114,8 \%)$.

\section{Ergebnisse}

Bei der Bestimmung der Vanillinmandelsäureausscheidung im Harn von 71 gesunden männlichen und weiblichen Probanden im Alter von 3-64 Jahren fanden wir im Mittel eine VMS-Ausscheidung von $4,1 \mathrm{mg} /$ 24 Stdn. Die niedrigste Ausscheidung zeigte ein 3-jähriges Kind mit 0,9 mg, die höchste ein 28jähriger Mann mit $8,6 \mathrm{mg}$ VMS/24 Stdn. Bei mehr als $90 \%$ der untersuchten Proben lag die Ausscheidung jedoch zwischen $1,5-7,0 \mathrm{mg} / 24$ Stdn. Bei 12 Patienten mit einem Phaeocbromocytom oder Neuroblastom fanden wir VMSWerte zwischen 10,8 und 142,3 (im Mittel 34,7) mg VMS/24 Stdn.

\section{Literatur}

1. Armstrong, M. D. und A. McMrluan, Federat. Proc. 16, 146 (1957). - 2. Armstrong, M. D., A. McMrllan und K. N. F. SHAw, Biochim. biophysica Acta (Amsterdam) 25, 422 (1957). 3. Axelrod, J., Science (Washington) 126, 400 (1957). - 4. AxeLROD, J., J. K. INSCOE, S. SENOH und B. WrTKOP, Biochim. biophysica Acta (Amsterdam) 27, 210 (1958). - 5. Axelrod, J., S. SENOH und B. WrTKOP, J. biol. Chemistry 233, 697 (1958). 6. Axelrod, J. und R. Tomchick, J. biol. Chemistry 233, 702 (1958). - 7. AxelroD, J., Science (Washington) 127, 754 (1958). - 8. Sourkes, T. L., Rev. canad. Biol. 17, 328 (1958). - 9. Goodall, McC., N. Krrshiner und L. Rosen, J. Clin. Invest. 38, 707 (1959). - 10. Kirshner, N., McC. Goodali und L. Rosen, Proc. Soc. exp. Biol. Med. 98, 627 (1958). - 11. Kraupp, O., H. Stormann, H. Bernsheimer und H. Obenaus, Klin. Wschr. 37, 77 (1959). - 12. Robinson, R., J. Ratcliffe und
J. SMITH, J. Clin. Path., London 12, 541 (1959). - 13. SUNDER ${ }^{-}$ MaN, F. W. jr., P. D. Cleveland, N. C. LaW und F. W. Sunder ${ }^{-}$ Man, Amer. J. Clin. Path. 34, 293 (1960). - 14. v. Studnitz, W., Scand. J. Clin. Laborat. Invest. 12, Suppl. 48 (1960). - 15. SANDLER, M. und C. R. J. Ruthven, Biochem. J. 80, 78 (1961). - 16. v. Studnitz, W. und A. Hanson, Scand. J. Clin. Laborat. Invest. 11, 101 (1959). - 17. v. Studnitz, W., Scand. J. Clin. Laborat. Invest. 11, 309 (1959). - 18. Grirlow, S. E., M. Mendlowitz, S. Khasts, G. Cohen und J. ShA, J. Clin. Invest. 39, 221 (1960). 19. Schmid, E. und N. Henning, Klin. Wschr. 41, 566 (1963). 20. Strobach, H., A. Beierbach und K. GreefF, NaunynSchmiedebergs Arch. exp. Pathol. Pharmakol. 251, 167 (1965). - 21. Greeff, K. und H. Strobach, Verh. Dtsch. Ges. inn. Med. 72, (1966). - 22. BRUNJES, S., N. England J. Med. 271, 120 (1964).

\section{Elektrophorese in horizontalem Polyacrylamidgel}

\section{Mitteilung: Die Trennung der Komponenten des Humanserums bei verscbiedenen Trennstrecken}

Von H. BIEL und O. ZwisLer

Aus der Bebringwerke A. G., Marburg

(Eingegangen am 17. August 1966)

Der Einfluß der Wanderungsstrēcke auf die Auftrennung von Humanserum und zweier Humanserumfraktionen bei Elektrophorese in horizontalem Polyacrylamid wurde untersucht.

The influence of the distance of migration in electrophoresis in horizontal polyacrylamide gel was studied on the fractionation of human serum and of two human serum fractions.

In einer vorhergehenden Arbeit $\left.{ }^{1}\right)$ untersuchten wir bei vorgegebener Trennstrecke (Abstand Auftragstelle Präalbumin etwa $25 \mathrm{~cm}$ ), inwieweit durch Änderung der Pufferzusammensetzung und -ionenstärke, der Gelkonzentration und dem Grad der Quervernetzung die Trennung der Komponenten des normalen Humanserums verbessert werden konnte. In der vorliegenden Arbeit soll gezeigt werden, welchen Einfluß die Wanderungsstrecke auf die Trennung der Serumproteine besitzt.

1) Zwisler, O. u. H. Biel diese Z. 4, 58 (1966).

\section{Methodik}

Testsubstanzen zur Elektrophorese

Normales Humanserum Hp 2-1, Gc 1-1 = „NMS“

Gesamt- $\alpha$-Fraktion eines normalen Humanserums Hp 1-1, gewonnen durch Elektrophorese in Polyvinylchlorid = „PVCE $\alpha-F_{r}$ "

$\alpha_{2}$-Fraktion eines normalen Humanserums Hp- 2-2, gewonnen durch zweimalige Elektrophorese in Polyvinylchlorid = „PVCE $\alpha_{2}-F^{e c}$

\section{Elektrophorese in PAA-Gel}

Cyanogum 41 wurde 5,5-proz. (g/Vol.) in Gelpuffer (0,0550 M Tris $-0,00646 \mathrm{M}$ Zitronensäure $-0,00268 \mathrm{M} \mathrm{NaOH}-0,00813 \mathrm{M}$ 